\title{
Long-Term X-ray Spectral Variability of Seyfert Galaxies with Swift
}

\section{S. D. Connolly*}

University of Southampton, Highfield, Southampton, SO17 1BJ, UK

E-mail: sdclg08@soton.ac.uk

\section{M. McHardy}

University of Southampton, Highfield, Southampton, SO17 1BJ, UK

E-mail: imh@astro.soton.ac.uk

\section{T. Dwelly}

Max-Planck-Institut für Extraterrestrische Physik, Giessenbachstrasse 1, 85748, Garching, DE

E-mail: dwelly@mpe.mpg.de

We present analysis of the long-term X-ray spectral variability of Seyfert galaxies as observed by Swift, which provides well-sampled observations over a much larger flux range and a much longer timescale than any other X-ray observatory. We examine long-term variability of three AGN: NGC 1365 (see Connolly et al. 2014), Mkn 335 and NGC 5548. At high fluxes, the 0.5-10 $\mathrm{keV}$ spectra soften with increasing flux, as seen previously within the $2-10 \mathrm{keV}$ band. However, at very low fluxes the sources also become very soft. We have fitted a number of models to the data and find that both intrinsic luminosity variability and variable absorption are required to explain the observations. In some systems, e.g. NGC 1365, the best explanation is a two-component wind model in which one component represents direct emission absorbed by a disc wind, with the absorbing column inversely proportional to the intrinsic luminosity, and the second component represents unabsorbed emission reflected from the wind. In other AGN the situation is more complex.

Swift: 10 Years of Discovery,

2-5 December 2014

La Sapienza University, Rome, Italy

\footnotetext{
*Speaker.
} 


\section{Introduction}

X-ray spectral observations have shown that many Seyfert galaxies possess absorbers whose absorbing columns vary by large amounts [1]. The detection of variability of the absorbing material on a timescales of hours has indicated that it must be close to the nucleus, at a distance similar to that of the Broad Emission Line Region [e.g. 2]; some absorption is claimed to be by clouds within the Broad Line Region, due to complete occultations which have been observed on timescales of days [3]. The existence of absorbing outflows in Seyferts, linked to disc winds, is also well established observationally [4]. Longer-term trends in the behaviour of these absorbers have, however, not been investigated in as much detail.

Here, we look at the long-term variability of three Seyfert galaxies - NGC 1365, NGC 5548 and Mkn 335 - which are all known to possess absorption variability. The three systems cover a range of masses, Eddington ratios and viewing angles (see Table 1).

\begin{tabular}{lccc}
\hline & Black Hole Mass $^{[4,5,6]}\left(\mathrm{M}_{\odot}\right)$ & ${\text { Eddington Ratio }\left(\mathrm{L}_{\text {Bol }} / \mathrm{L}_{\text {Edd }}\right)^{[7]}}$ & Viewing angle $^{[8]}\left({ }^{\circ}\right)$ \\
\hline NGC 1365 & $2.9 \times 10^{7}$ & 0.02 & $36-60$ \\
\hline NGC 5548 & $6.5 \times 10^{7}$ & 0.04 & $22-40$ \\
\hline Mkn 335 & $2.5 \times 10^{7}$ & 0.38 & $38-49$ \\
\hline
\end{tabular}

Table 1: Physical parameters of each of the three Seyfert galaxy nuclei.

\section{Observations \& Data Reduction}

The entire set of Swift XRT data available on the HEASARC archive ${ }^{1}$ for each source was used for this study. For each source, several hundred spectra from individual Swift 'visits', or exposures, were used, each with a total of several hundred kiloseconds of exposure time. A small fraction of the spectra were rejected due to very low signal to noise or artifacts near the source.

The data were reduced with an automatic pipeline which uses the XRTPIPELINE software, previously employed in e.g. [5]. The XSELECT tool was used to extract spectra and lightcurves, using flux-dependent source and background extraction regions chosen such that background contamination was reduced at faint fluxes and the effects of pile-up were mitigated at high fluxes. The X-ray background was subtracted using a background annulus region. The observed XRT count rates were then corrected for bad pixels, vignetting effects, and the finite extraction aperture.

Fig. 1 shows a lightcurve of all of the Swift data over the nine-year period of observation.

\section{Data Analysis}

\subsection{Spectral Hardness}

Fig. 2 shows the hardness ratio $(\mathrm{H}+\mathrm{S} / \mathrm{H}-\mathrm{S})$ against the hard count rate of each source. Here, we look at spectral variations within the $0.5-10.0 \mathrm{keV}$ band, a broader band than the $2.0-10.0 \mathrm{keV}$ energy band on which most previous studies concentrate. As the low-energy end of the spectrum is more sensitive to absorption, this energy range enables a more complete look at spectral changes due to absorption. In each case, hard and soft emission are defined as $2.0-10.0 \mathrm{keV}(\mathrm{H})$ and $0.5-2.0 \mathrm{keV}(\mathrm{S})$, respectively.

The hardness ratios all show similarities - all three display softening with increasing flux at higher flux levels. NGC 1365 also becomes extremely soft at the lowest flux levels, a trend also

\footnotetext{
${ }^{1}$ http://heasarc.gsfc.nasa.gov/cgi-bin/W3Browse/swift.pl
} 


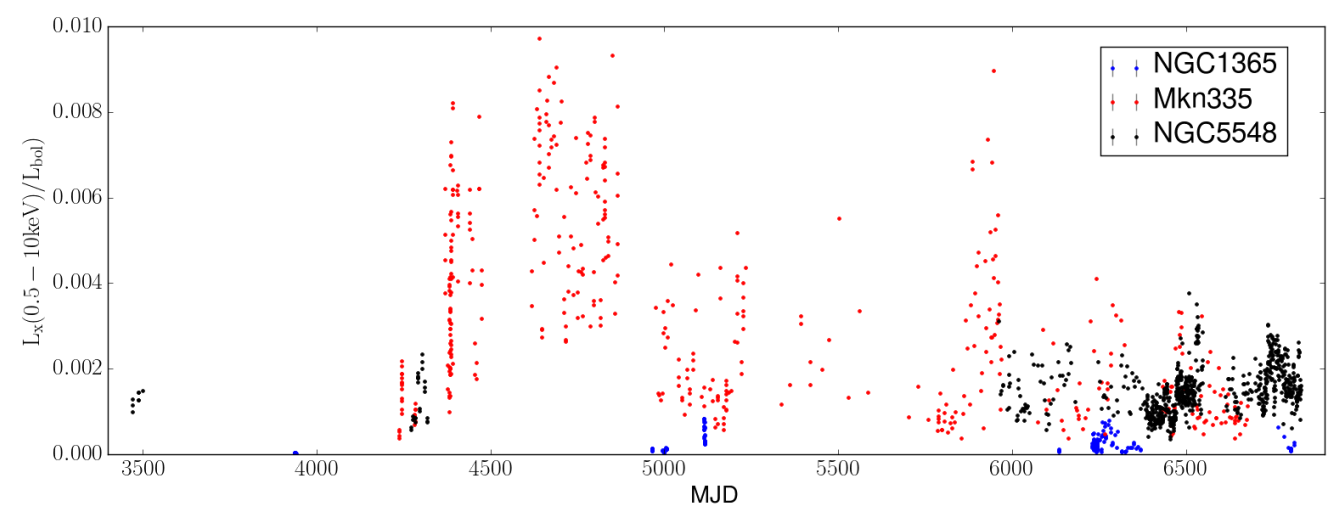

Figure 1: The ratio of $0.5-10 \mathrm{keV}$ X-ray luminosity to Eddington luminosity of each source, as a proxy for the Eddington ratio.

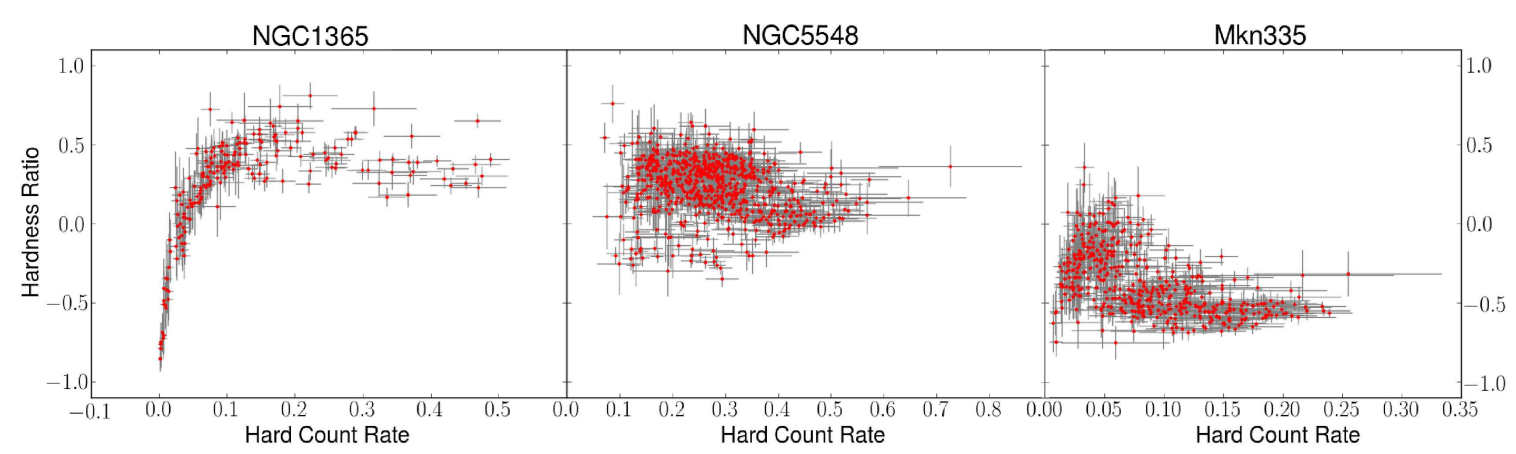

Figure 2: The hardness ratio $(\mathrm{H}-\mathrm{S} / \mathrm{H}+\mathrm{S})$ against hard count rate for each source.

hinted at by the Mkn 335 data, but not seen in NGC 5548. Broadly, the Mkn 335 data are, however, more similar to those of NGC 5548, with a much broader range of hardnesses at a given flux.

NGC 1365 shows a very small amount of scatter in hardness, implying that the spectral shape is fairly constant at a given flux level. The spectra of NGC 1365 could therefore be binned by flux, giving a high signal-to-noise ratio spectrum at each flux level. As the Mkn 335 and NGC 5548 data show much greater variation in hardness at a given flux, they were instead binned in time intervals.

\subsection{Spectral Modelling}

The binned spectra of each source were fitted with two possible absorption models - a partial covering model and a two-component model consisting of two power laws, one of which is absorbed by an ionised absorbing column (e.g. Fig 3). Table 2 shows the reduced $\chi^{2}$ values for variants of each model. Both models have previously been used to describe AGN spectra, e.g. NGC 4945 [6] and NGC 1365 [7]. In all fits, the underlying power law spectral index is tied, such that it remains constant between spectra. In reality, $\Gamma$ is likely to vary slightly, and allowing it to vary does give better fits. However, due to a degeneracy with varying absorption, a large, unphysical range of $\Gamma$ is produced.

For all three sources, partial covering models only provided a better fit when the covering fraction was free to vary - as this makes the model equivalent to the two-component, the latter was 


\begin{tabular}{|c||c|c|c||c|c|c|}
\hline \multicolumn{1}{|c||}{} & \multicolumn{2}{c}{ Absorbed \& unabsorbed powerlaws } & \multicolumn{3}{c}{ Partial Covering } \\
\cline { 2 - 7 } & All free & $\mathrm{N}_{\mathrm{H}}$ tied & Ionisation Tied & All free & $\mathrm{N}_{\mathrm{H}}$ tied & Covering fraction tied \\
\hline NGC 1365 & 1.39 & 2.28 & 1.42 & 1.45 & 2.57 & 1.45 \\
\hline NGC 5548 & 1.03 & 1.13 & 1.04 & 1.06 & 1.14 & 1.09 \\
\hline Mkn 335 & 1.12 & 1.13 & 1.14 & 1.24 & 1.23 & 1.35 \\
\hline
\end{tabular}

Table 2: Total reduced $\chi^{2}$ values of variations of two absorption models, fitted simultaneously to the total sample of binned spectra of each source.

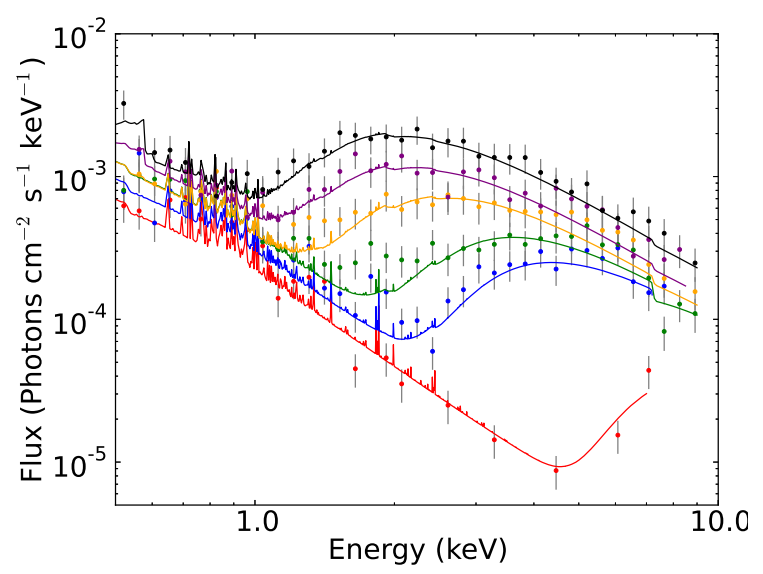

Figure 3: Sample of the flux-binned spectra of NGC 1365, simultaneously fitted with the best-fitting model, consisting of two power laws, one of which is absorbed by a partially ionised absorbing column.

used in subsequent analysis. The best-fit parameters of this model showed that the variability of NGC 1365 requires a decrease in absorbing column with increasing flux, regardless of whether or not the ionisation state was allowed to vary - changes in the ionisation state of the absorber alone were found to be unable to account for the observed spectral variability. When both the absorbing column and ionisation state were allowed to vary freely, only small changes in the ionisation were required by the best-fitting model and the inverse relationship between the absorbing column and flux was still present. In Mkn 335 and NGC 5548, however, changes in ionisation state and absorbing column alone described the spectral variability equally well, meaning neither source of variability could be ruled out.

\section{Discussion}

\subsection{A Possible Relationship Between Source Flux and Column Density}

Fig. 4 (left) shows a clear decrease in the absorbing column as the normalisation of the absorbed power law increases in the two-component model of NGC 1365. As this component dominates the unabsorbed luminosity, the absorbing column is therefore inversely proportional to the source luminosity. Whilst a reduction in absorption with increasing flux might initially be assumed to be due to increased ionisation, spectral models involving varying ionisation alone do not fit the data. Fits to spectra of NGC 4151 at different flux levels show a similar reduction in the absorbing column with increasing flux [8], implying that this relationship is not unique to NGC 1365. 

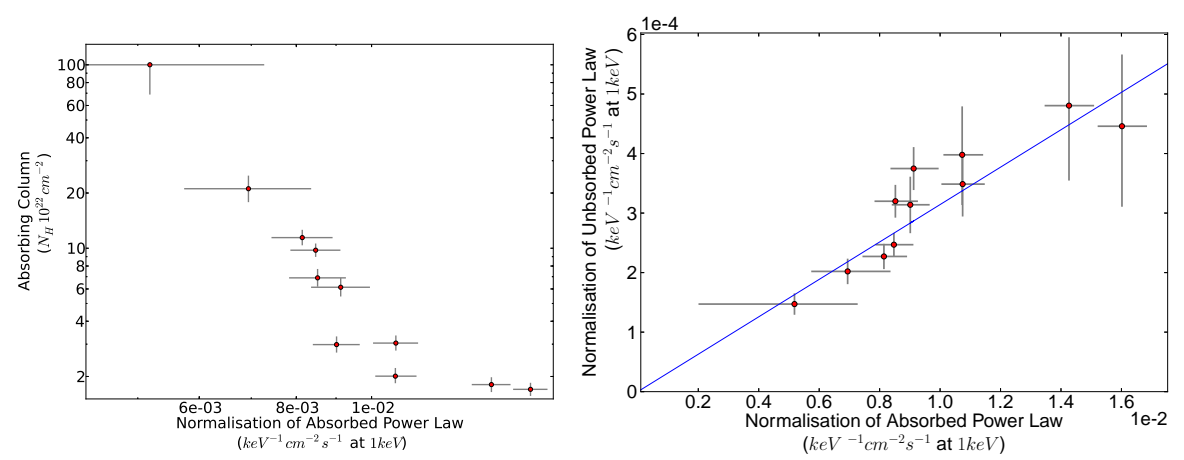

Figure 4: Plots of the parameters of the two-component model of NGC 1365. (Left) The normalisation parameter of the absorbed power law against the column of the absorbing material. (Right) The normalisation parameter of the absorbed power law against that of the unabsorbed power law, fitted with a linear model.

For the same model, Fig. 4 (right) shows the normalisation of the unabsorbed power law to be well correlated with that of the absorbed power law $(r=0.91)$, implying a common origin. The underlying luminosity of the source is also shown to be changing; the observed changes in flux are therefore not solely due to changes in the absorbing medium.

A similar inverse relationship between the absorbing column and source luminosity is seen in the two-component model of Mkn 335 in which the ionisation state is not allowed to vary. However, the data are equally well fit when the ionisation state alone is allowed to vary, meaning the inverse relationship cannot be confirmed. The spectra of NGC 5548 approximately equally well fit whether of not the ionisation state is tied, however there is no clear correlation between the absorbing column and the luminosity, implying that the variablility is not caused by the same mechanism as NGC 1365. Both NGC 5548 and Mkn 335 also show underlying continuum variability in addition to absorption variation.

Although unlikely to explain the absorption variability of all AGN, the spectral variability displayed by at least one AGN can be entirely explained by systematic variation of the absorber, with the absorption varying inversely with luminosity.

\subsection{AGN Wind Model}

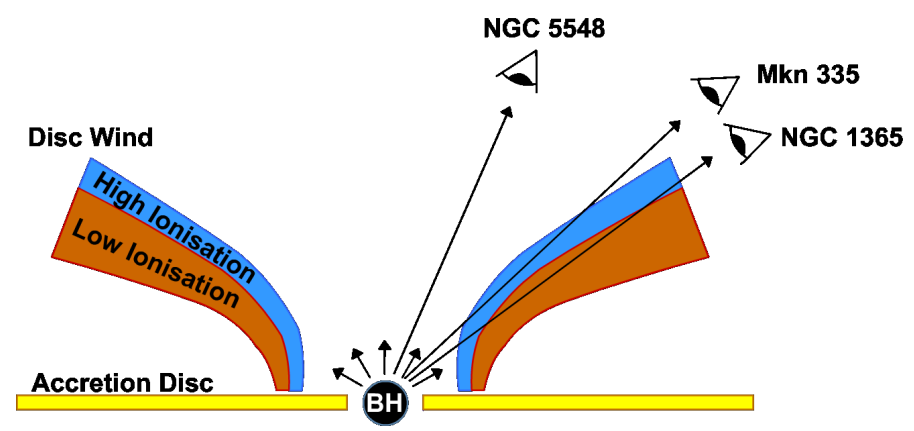

Figure 5: Possible orientations of each AGN with respect to a disc wind. As in the (author?) [11] wind model.

Absorption in AGN X-ray spectra is often attributed to disc winds (see e.g. [9], [4]). Both models and single-epoch data samples suggest that a rise in accretion rate, and therefore an increase 
in X-ray luminosity, naturally leads both to an increase in the radii from which the wind arises, and to an increase in the opening angle of the wind $[10,11,4]$. Thus, if the observer views the X-ray source at an inclination such that they are looking through the inner edge of the wind, an increase in accretion rate would move the wind out of the line of sight of the observer, lowering the measured absorbing column. This will thus naturally lead to the inverse relationship between absorbing column and luminosity which we see clearly in NGC 1365. If Mkn 335 is observed through the very inner part of the wind, such that ionisation effects are greater, whilst our view of NGC 5548 is unobscured (see Fig. 5), this model has the potential to explain the variability of all three AGN.

\section{Conclusions}

The Swift spectra of NGC 1365, Mkn 335 and NGC 5548 show large spectral variation, a significant cause of which is found to be changes in the absorbing material. In NGC 1365, the absorbing column decreases with increasing X-ray flux, which could be explained by a varying disc wind. The Mkn 335 data hints at similar behaviour at lower fluxes, whilst the variation of NGC 5548 is best explained by changes in the ionisation state of the absorber.

\section{Acknowledgments}

SDC thanks the STFC for support under a studentship and IMcH thanks the STFC for support via grant ST/G003084/1.

\section{References}

[1] Risaliti, G., Elvis, M. and Nicastro, F., Ubiquitous variability of X-ray absorbing column densities in Seyfert 2 Galaxies, ApJ, 571 (2002) 234.

[2] Puccetti, S., Fiore, F., Risaliti, G., Capalbi, M., Elvis, M. and Nicastro, F., Rapid nH changes in NGC 4151, MNRAS, 377 (2007) 607.

[3] Risaliti, G., X-ray observations of NGC 1365: time-resolved eclipse of the X-ray source, ASPC, 373 (2007) 1.

[4] Tombesi, F. et al., Unification of X-ray winds in Seyfert galaxies : from ultra-fast outflows to warm absorbers, MNRAS, 430 (2013) 1102.

[5] Connolly, S. D., McHardy, I. M. and Dwelly, T., Long Term Wind-Driven X-Ray Spectral Variability of NGC 1365 with Swift, MNRAS, 440 (2014) 3503.

[6] Done, C., Madejski, G. and Smith, D., NGC 4945: The Brightest Seyfert 2 Galaxy at 100 keV, ApJ, 463 (2014) 63.

[7] Risaliti, G. et al., The XMM-Newton long look of NGC 1365: uncovering of the obscured $X$-ray source, MNRAS, 393 (2009) 1.

[8] Lubiński, P. et al., Extreme flux states of NGC 4151 observed with INTEGRAL, MNRAS, 408 (2010) 1851.

[9] Kaastra, J. S. et al., X-ray absorption lines in the Seyfert 1 galaxy NGC 5548 discovered with Chandra-LETGS, A\&A, 354 (2000) 83.

[10] Nicastro, F., Broad Emission Line Regions in Active Galactic Nuclei: The Link with the Accretion Power, ApJ, 530 (2000) 65.

[11] Elvis, M., A Structure for Quasars, ApJ, 545 (2000) 76. 